\title{
Ce que le moustique nous apprend sur le dualisme anthropocentrisme/biocentrisme : perspective interdisciplinaire sociologie/biologie
}

\author{
Cécilia Claeys ${ }^{1}$, Julien Sérandour ${ }^{2}$ \\ 1 Sociologue, UMR6012 ESPACE, Laboratoire DESMID, Université de la Méditerranée, 163 avenue de Luminy, \\ 13288 Marseille cedex 9, France \\ 2 Biologiste, UMR5553, Laboratoire d'écologie alpine, Université Joseph Fourier, BP 53, 38041 Grenoble cedex 9, France
}

\begin{abstract}
D'une société où l'homme est le maître et l'ordonnateur de la nature (anthropocentrisme) une transformation s'opère vers une situation où il se voit comme un élément, parmi d'autres, d'un environnement complexe (bio-centrisme). Ses responsabilités changent; il se sent comptable de lui-même comme du milieu où il vit. Cet article analyse expérimentalement cette évolution dans le cas de la cohabitation des moustiques et des hommes dans le delta du Rhône et les vallées alpines. Les auteurs constatent que cette cohabitation est parfois volontairement décidée, parfois seulement tolérée, parfois enfin difficilement subie. De l'anthropocentrisme au bio-centrisme existent des itinéraires différents émaillés d'hésitations, d'avancées et de reculs.
\end{abstract}

La Rédaction

\section{Mots-clés :}

moustique; interdisciplinarité ; sociologie ; biologie ; zones humides

Keywords:

Mosquito, interdisciplinarity, sociology, biology, wetlands

\begin{abstract}
Résumé - Cet article propose une analyse interdisciplinaire sociologie/biologie du rapport au moustique et à la démoustication. Deux terrains sont étudiés, le delta du Rhône et les vallées alpines. Rappelant l'héritage anthropocentrique de nos sociétés, l'analyse montre comment les récentes évolutions des discours et des pratiques vis-à-vis du moustique tendent vers un référentiel biocentrique. Ce glissement prend la forme d'articulations entre savoirs scientifiques et vernaculaires, préoccupations environnementales, sanitaires et socioéconomiques. Une première partie analyse les interrelations sociales et biologiques entre l'insecte et son territoire. Une deuxième partie met en regard les persistances anthropocentriques et les référentiels biocentriques en développement. Une dernière partie analyse les «acrobaties taxinomiques » à l'œuvre et leur traduction en termes de contrôle de la nuisance.
\end{abstract}

\begin{abstract}
Anthropocentrism/biocentrism: learning from mosquitoes, an interdisciplinary sociology-biology perspective. Based on an interdisciplinary analysis associating sociology and biology, this paper studies the social discourses and practices concerning mosquitoes and mosquito pest management in France. The qualitative and quantitative sociological field studies involved two areas, the Rhone river delta and the French alpine valleys. Recalling the anthropocentric heritage of our western societies, our paper shows an evolution, linked to recent social changes, towards more biocentric references, a fact that is noticeable in discourses and attitudes toward mosquitoes. Rather than a radical switch, processes of cohabitation and hybridizing may be observed, combining popular and scientific knowledge, environmental, medical and social issues. The first part of this paper highlights the social and biological relationships between the insect and its habitat, i.e., wetlands. The second part focuses on the forms of articulation between anthropocentrism and biocentrism, especially through the dualism pest/useful insect. The third and last part analyses how the "taxonomic arrangements" between anthropocentrism and biocentrism have triggered an evolution of French public policies regarding mosquito pest management.
\end{abstract}

Auteur correspondant :

C. Claeys, cecilia.claeys@univmed.fr 


\section{Le moustique, entre biologie et sociologie}

Cet article propose une analyse interdisciplinaire $\mathrm{du}$ moustique, être vivant mu par des principes biologiques qui l'amènent à faire de l'être humain l'un de ses hôtes, ce dernier déployant en retour des représentations et des pratiques sociales spécifiques vis-à-vis de cet insecte piqueur ${ }^{1}$. La biologie nourrit la réflexion par sa connaissance du moustique et de son habitat en tant que matérialité et la sociologie fournit une compréhension des représentations et des pratiques relatives à cet insecte, en les resituant dans l'analyse du rapport à la nature et au territoire. Il s'agit moins de confronter la subjectivité des acteurs sociaux aux « vérités » scientifiques que de saisir l'imbrication entre processus biologiques et sociaux.

Cet article s'appuie sur plusieurs enquêtes de terrain réalisées en France métropolitaine entre 1995 et 2006. Initié dans le delta du Rhône ${ }^{2}$, le premier volet empirique est constitué d'un corpus de 60 entretiens semi-directifs réalisés auprès des acteurs socioéconomiques et de riverains (de 1995 à 2002), adoptant comme méthode d'échantillon le principe de la saturation des discours. Le deuxième volet est composé de deux campagnes d'enquête par questionnaires (2001 et 2002), l'une auprès d'un échantillon représentatif des habitants de la Camargue et de la ville d'Arles (communes d'Arles et des Saintes-Maries-de-laMer, soit 160 individus interrogés), l'autre auprès d'un échantillon représentatif des habitants de la Petite Camargue (commune d'Aigues-Mortes, soit 100 individus interrogés). Le dernier et plus récent des volets concerne les enquêtes réalisées en région Rhône-Alpes, en 2004 et 2005. Il s'agit d'une transposition et d'une adaptation du protocole d'enquête préalablement conçu en Camargue. L'accent est mis sur l'approche quantitative, avec la réalisation d'une enquête par questionnaires auprès d'un échantillon représentatif de la population rhône-alpine (319 individus interrogés). Le corpus qualitatif se limite à une phase de préenquête (une dizaine d'entretiens semi-directifs auprès d'élus, d'agriculteurs, de professionnels du tourisme et du domaine médical ${ }^{3}$. Pour les trois

\footnotetext{
${ }^{1}$ Nous remercions l'Entente interdépartementale pour la démoustication (EID) Ain, Isère, Rhône, Savoie pour son soutien technique et financier à cette étude, ainsi que le $\mathrm{D}^{\mathrm{r}}$ Muriel Raveton et le $\mathrm{P}^{\mathrm{r}}$ Guy Lempérière pour la relecture.

2 Les enquêtes relatives au delta du Rhône ont pu être réalisées grâce au soutien financier du ministère de l'Environnement, du Parc naturel régional de Camargue et de l'EID Méditerranée. L'idée initiale de développer une "sociologie du moustique et de la démoustication » nous fut donnée par Bernard Picon. Qu'ils en soient tous ici remerciés.

${ }^{3}$ Ces différentes enquêtes ont pu être menées grâce au soutien financier du ministère de l'Environnement (programme national de recherche Dynamiques associatives et cadre de vie), du Parc naturel régional de Camargue et de l'EID Méditerranée, dans le cadre du programme LIFE Environnement (LIFE 99env/F/000489), ainsi que de l'EID Ain, Isère, Rhône, Savoie.
}

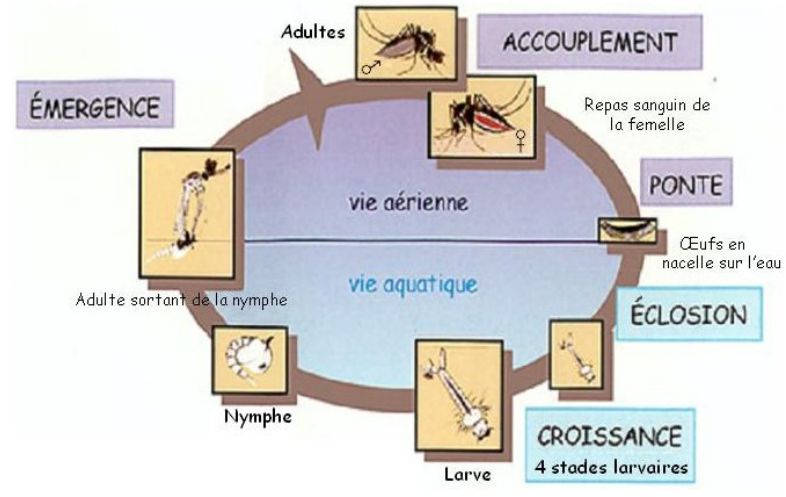

Fig. 1. Cycle de vie du moustique commun Culex (Culex) pipiens (Linnaeus). (Source : EID Ain, Isère, Rhône, Savoie.)

terrains, il s'agit de questionnaires téléphoniques. Les critères de construction des échantillons sont l'âge, le sexe et la classe sociale des individus. Les données sont soumises à un traitement informatique (logiciels Modalisa ${ }^{\circ}$ et Sphinx Plus ${ }^{2} \odot$ ) fondé sur les statistiques descriptives classiques (test de corrélation du khi2).

Ces trois terrains se différencient selon deux axes. En premier lieu, Petite Camargue et vallées alpines sont des zones démoustiquées, tandis que la Camargue au sens strict (entre les deux bras du Rhône) ne l'est pas. En second lieu, la Camargue est depuis plus de dix ans le théâtre d'une controverse locale relative à sa démoustication, tandis qu'en Petite Camargue et en Rhône-Alpes, la démoustication ne fait pas ou peu controverse.

Cet article est structuré en trois parties. Dans un premier temps, une analyse diachronique du traitement social du moustique et de son milieu est proposée, en y introduisant les connaissances biologiques relatives à cet insecte. En un deuxième temps, l'accent est mis sur la pluralité des représentations et des pratiques contemporaines vis-à-vis de cet animal, qu'il convient de situer plus largement dans la question du rapport entre l'homme et la nature. Enfin, une dernière partie souligne comment ces représentations sociales plurielles façonnent des demandes sociales paradoxales, auxquelles les politiques publiques tentent de répondre.

\section{De l'anthropocentrisme au biocentrisme : dynamique sociale et naturelle d'un insecte et de son territoire}

Les moustiques, ou Culicidés, sont des insectes faisant partie de l'ordre des Diptères (une seule paire d'ailes). On dénombrait 63 espèces de moustiques connus à la fin du $X X^{e}$ siècle en France (incluant la Corse), mais la liste évolue rapidement (Schaffner, 1998). Les œufs de moustiques éclosent dans l'eau pour donner des larves puis des nymphes aquatiques; ensuite, l'émergence survient, favorisée par le réchauffement de l'eau (Fig. 1). 


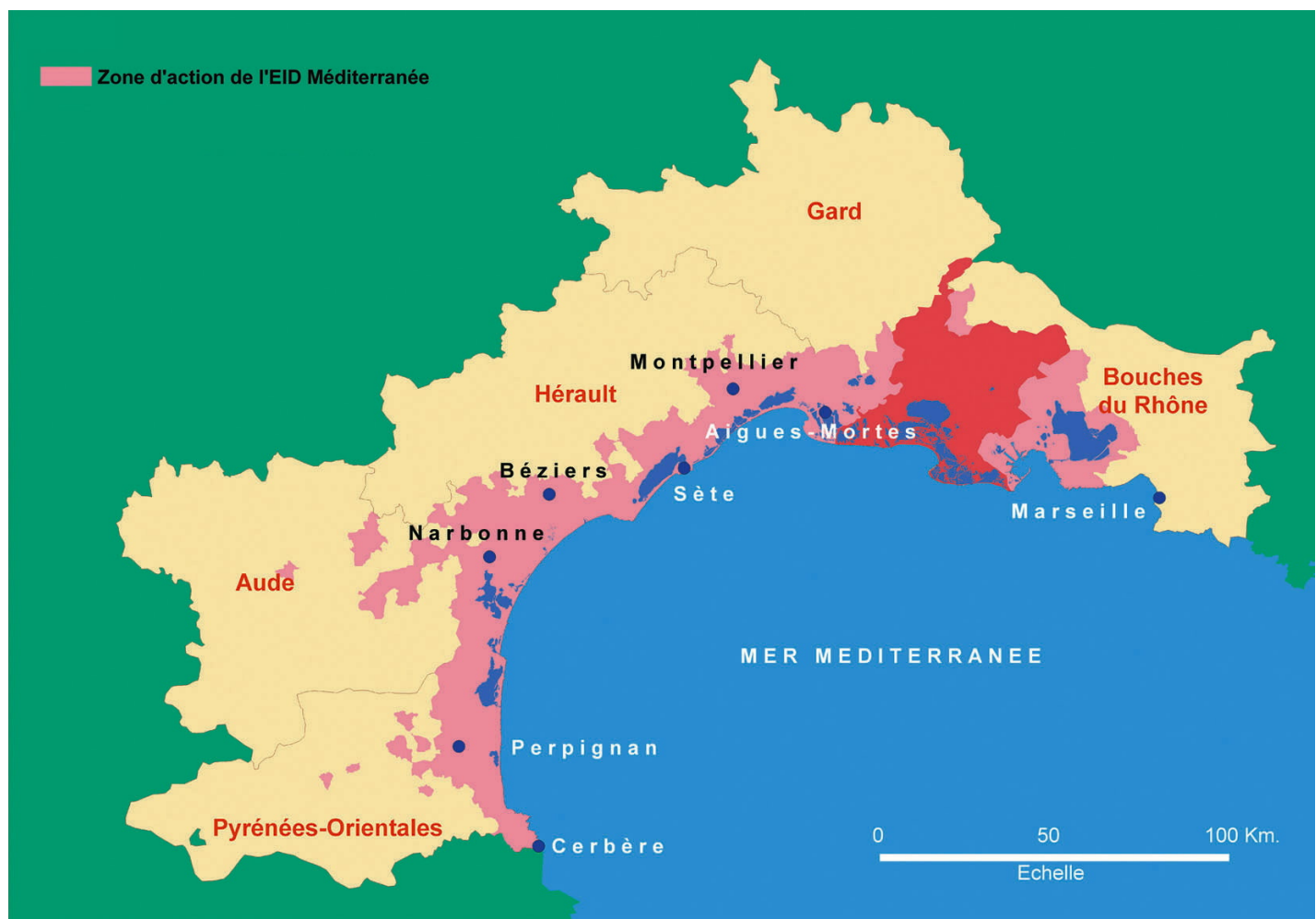

Fig. 2. Les zones d'activité de l’EID Méditerranée. (Source : EID Méditerranée.)

Les adultes sont aériens et vivent dans les milieux frais et humides, avec une végétation abondante car ils se nourrissent de sève et de nectar. Après l'accouplement, les femelles recherchent un hôte pour effectuer un repas de sang nécessaire à la maturation des ovocytes, qui, après fécondation, permettent le développement des œufs. Le développement du moustique s'arrête l'hiver pendant une période de diapause, qui intervient au stade des œufs (Aedes) ou des larves (Coquillettidia), en fonction de l'espèce. Tous les moustiques ne sont pas anthropophiles (piquant l'homme), leur spectre d'hôtes s'étend à l'ensemble des vertébrés, même si la plupart des espèces montrent une préférence trophique pour un groupe taxinomique particulier (oiseaux, mammifères...). Le moustique perfore la peau de ses stylets, qui pénètrent dans les capillaires sanguins. La prise de sang est précédée d'une injection sous-cutanée de salive contenant un anticoagulant; $c^{\prime}$ est par ce biais que peuvent être transmis des agents pathogènes. C'est aussi cette injection de salive qui provoque chez l'hôte des réactions cutanées plus ou moins fortes (Rodhain et Perez, 1985).

Le moustique, inféodé aux zones humides, partage l'histoire de ces milieux. Territoires marginaux, abandonnés aux gens de peu, les marais sont, jusqu'au début du $X X$ e siècle, associés aux miasmes, à la puanteur et aux maladies (Bata et al., 2002 ; Corbin, 1986). Paludisme, fièvres et infections touchaient en effet les habitants et usagers de ces zones humides (Bata et al., op. cit. ; Sérandour et al., 2007).
Les «gens des marais » constituent les parents pauvres d'une société longtemps agricole, marquée en profondeur par une culture anthropocentrique. L'anthropocentrisme appréhende la nature comme une ressource au service de l'homme, qui se doit de la prélever, la domestiquer, la conquérir, transformer en utile (pour lui) ce qui ne l'est pas, détruire ce qui constitue un danger (réel ou perçu) et qui ne peut être soumis à son contrôle. De ce point de vue, le beau et le bien désignent ce qui est cultivé, domestiqué, utile à l'homme. Dans ce contexte, les marais français sont assainis dans une logique sanitaire et de mise en valeur agricole, lentement d'abord, parce que de façon manuelle, puis avec l'efficacité de la modernité montante du XIX ${ }^{\mathrm{e}}$ siècle. Cette modernité triomphante a toutefois négligé quelques marais, par désintérêt pour des espaces potentiellement peu rentables en termes de mise en valeur agricole, ou bien pour les utiliser comme champs d'expansion dans la gestion des crues, afin de protéger villes et zones industrielles des inondations, parfois aussi pour ne pas avoir pu s'imposer face à de fortes oppositions locales. Les deux territoires étudiés ici ont en commun d'avoir traversé le XIX ${ }^{\mathrm{e}}$ siècle en conservant une partie de leurs zones humides, qui bénéficient aujourd'hui des politiques de protection de la nature.

Les vallées alpines ont subi de profondes transformations depuis le XIX ${ }^{\mathrm{e}}$ siècle, avec des changements dans les pratiques agricoles, l'urbanisation et l'assainissement des zones humides. Pourtant, certaines subsistent, comme les marais associés au lac du Bourget ou quelques marais des 
plaines alluviales alpines, qui sont protégées par de nombreux outils juridiques (directives européennes Habitat et Oiseaux, $\mathrm{ZNIEFF}^{4}$, statut de réserve naturelle).

En Camargue, les ingénieurs des Ponts et Chaussées y virent un territoire de prédilection pour la mise en œuvre de grands travaux de drainage des marais (Allard, 1999). Cependant, une résistance locale vit le jour, s'opposant à ces projets et au mouvement d'uniformisation du territoire dont ces derniers étaient porteurs (Picon, 1978). Cette résistance locale a façonné ce territoire, qui demeure aujourd'hui encore marqué par une identité territoriale forte (Picon, op. cit. ; Pelen, 1987; Claeys-Mekdade, 2000 et 2003 ; Claeys-Mekdade et al., 2002). La Camargue, par ses zones humides, devient au cours du $X^{\mathrm{e}}$ siècle un haut lieu de protection de la nature, où se concentrent les attentions des politiques environnementales émergeant sous leur forme contemporaine à partir de la fin des années $1960^{5}$

Ces deux territoires traversèrent donc le $\mathrm{XIX}^{\mathrm{e}}$ siècle en conservant leurs zones humides et, de façon corollaire, leurs moustiques. Puis, grâce ou à cause de l'industrie chimique, engrais et pesticides font leur apparition pour lutter contre les champignons (fongicides), les mauvaises herbes (herbicides), les insectes ravageurs de cultures, vecteurs de maladies ou nuisibles (insecticides). Le développement des insecticides rendit techniquement possible, notamment, le développement de politiques de démoustication des territoires à grande échelle.

Considérée par les acteurs politiques comme une condition sine qua non du développement économique, la démoustication des zones à vocation touristique et résidentielle ${ }^{6}$ est confiée à des organismes spécialisés, les Ententes interdépartementales de démoustication (EID), créées au début des années 1960. Ces politiques de démoustication font, à ce titre, intégralement partie des politiques d'aménagement du territoire.

Le Languedoc-Roussillon, touristique et résidentiel, ainsi que le pourtour de l'étang de Berre, industriel et résidentiel, sont démoustiqués par l'EID Méditerranée, tandis que la Camargue, haut lieu de protection de la nature, n'est pas soumise à cette politique de démoustication (Fig. 2). En revanche, les vallées alpines sont démoustiquées par l'EID Ain, Isère, Rhône, Savoie, que ce soit en zones touristiques, résidentielles, industrielles ou naturelles (Fig. 3). Tout en étant au cœur du territoire démoustiqué, le lac du Bourget représente à la fois un fragile réservoir de biodiversité et un attrait pour la moitié

\footnotetext{
${ }^{4}$ Zones naturelles d'intérêt écologique faunistique et floristique.

${ }^{5}$ La Réserve naturelle de Camargue est créée dés 1927, le Parc naturel régional de Camargue (PNRC) en 1972, le delta s'inscrit dans la convention internationale ce Ramsar depuis 1986, le Conservatoire du littoral y acquiert des terrains depuis 1977.

${ }^{6}$ Vocation résidentielle en tant que corollaire au développement économique, qui attire une main-d'œuvre nouvelle.
}

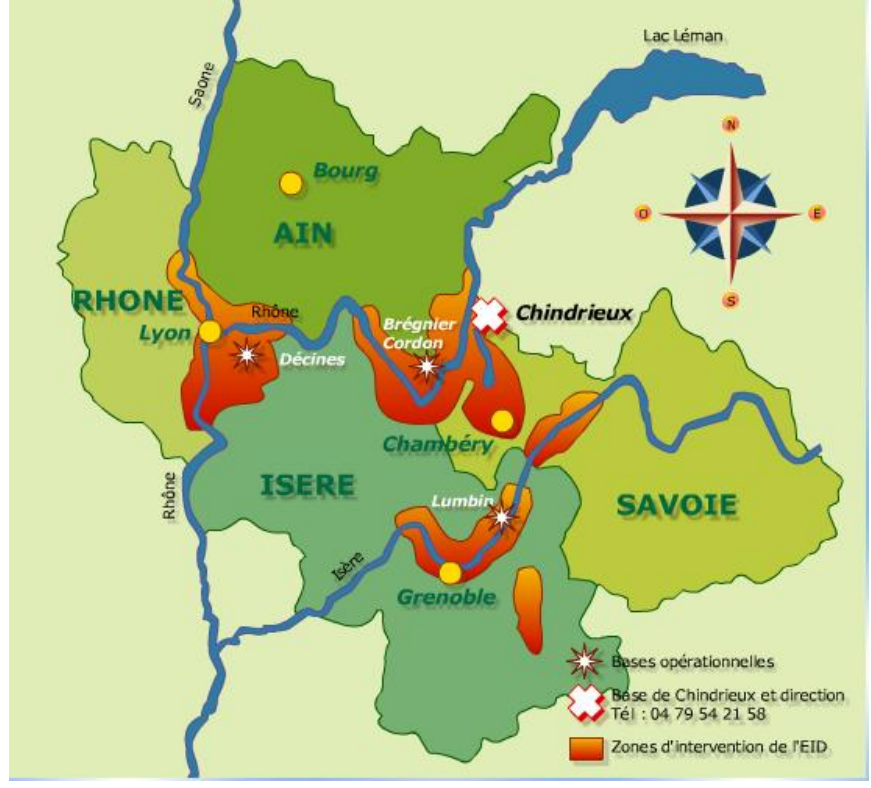

Fig. 3. Les zones d'activité de l'EID-Rhône Alpes. (Source : EID Ain, Isère, Rhône, Savoie.)

de la population savoyarde qui vit, travaille et se déplace autour de ce lac.

Ainsi, 1'héritage anthropocentrique cède du terrain au biocentrisme, cette conception du monde plaçant la nature au cœur de ses préoccupations, rejetant l'homme au rang de destructeur, de pollueur, prônant la protection des écosystèmes, de leur biomasse et de leur biodiversité. De ce point de vue, le bien et le beau désignent ce qui est sauvage, naturel, ou tout au moins perçu comme tel. L'espace rural, hier lieu de production, est redéfini comme lieu de ré/recréation par et pour des urbains en mal de nature (Picon, 1978 ; Chapuis et al., 2001). Dans ce contexte, les marais, hier désignés comme des espaces putrides à assainir, sont aujourd'hui considérés comme des espaces «naturels » à protéger, ainsi que le stipule notamment la convention de Ramsar adoptée dès 1971, traité intergouvernemental rejoint par la France en 1986.

\section{Le moustique, utile ou nuisible? Une rencontre entre anthropocentrisme et biocentrisme}

Du marais à assainir à l'écosystème à protéger, un passage s'opère du sale vers le propre, à travers ses dimensions "idéelles et matérielles ${ }^{7}$ ». Le matériel, c'est l'ensemble des fonctions remplies par les zones humides, d'un point de vue écosystémique. Considérées comme les habitats les plus productifs sur Terre, les zones humides sont aussi des réserves de biodiversité, car plus de $50 \%$ des espèces d'oiseaux en dépendent et $30 \%$ des espèces

\footnotetext{
7 Pour reprendre la formule de Godelier (1984).
} 


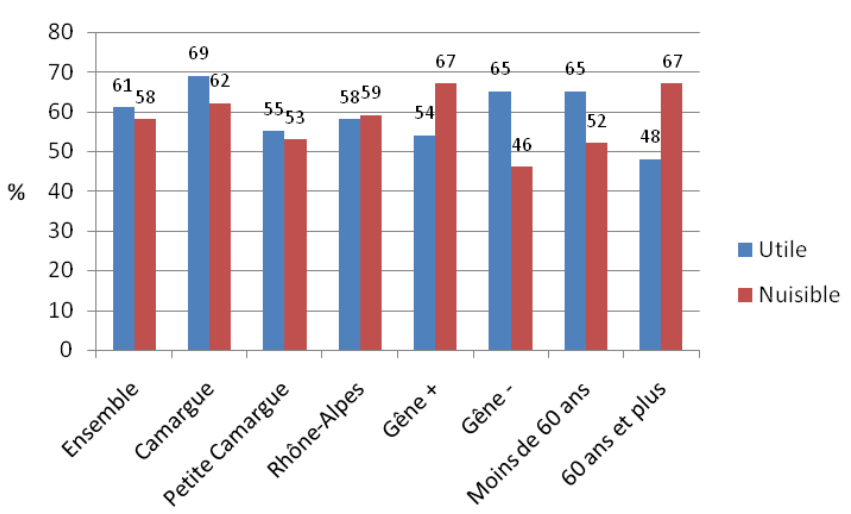

Fig. 4. Les effets territoriaux, cognitifs (sentiment de gêne) et démographiques (âge) sur la qualification du moustique : utile/nuisible.

végétales remarquables et menacées en France y sont inféodées $\left(\mathrm{IFEN}^{8}\right)$. Les conditions écologiques variées des zones humides offrent une niche à une diversité d'espèces, pour la plupart menacées, diversité dont font partie les moustiques (Willott, 2004). L'idéel, c'est la redéfinition des zones humides comme culturellement désirables, en tant que "nature sauvage » à préserver et paysages à admirer (Bata et al., 2002). Ce qui était considéré comme une souillure indésirable est redéfini comme une richesse aux qualités biologiques et esthétiques culturellement valorisées. L'idéel, c'est aussi le doute jeté sur le statut de la faune caractéristique de ce milieu. Tout comme son habitat, hier culturellement et socialement indésirable, aujourd'hui désiré, le moustique passe-t-il du statut de nuisible à celui d'utile?

Lors des enquêtes par questionnaires, deux questions étaient à ce titre posées : " Estimez-vous que le moustique est un insecte nuisible? ? et "Selon vous, le moustique a-t-il une utilité? » Il se trouve que la majorité de l'échantillon répond positivement aux deux questions (Fig. 4). En effet, parmi les réponses recueillies, les proportions sont proches entre la part des individus qualifiant le moustique de nuisible et ceux le qualifiant d'utile, avec respectivement $58 \%$ et $61 \%$ de l'ensemble de l'échantillon ${ }^{9}$. Les trois points d'écart en faveur du qualificatif « utile» ne permettent pas de dégager une posture véritablement dominante. L'autre point remarquable est l'émergence de la figure paradoxale du moustique « utile/nuisible », portée par prêt d'un tiers des personnes interrogées, qui associent en effet ces deux qualificatifs au même insecte.

Il convient de souligner que les habitants de la zone non démoustiquée (la Camargue au sens strict) sont plus enclins que les autres à qualifier le moustique de nuisible.

\footnotetext{
${ }^{8}$ Consulter le site de l'Institut français de l'environnement (IFEN) sur les zones humides pour un complément d'informations : http://www.ifen.fr/zoneshumides/

9 Par « ensemble de l'échantillon », il faut entendre les trois terrains étudiés : Camargue, Petite Camargue et Rhône-Alpes.
}

À l'effet objectivable d'exposition des individus aux piqûres, s'ajoutent des considérations sociales et cognitives. Quel que soit le terrain, l'expression d'un sentiment de gêne amène davantage les individus à qualifier le moustique de nuisible, et, réciproquement, un sentiment de gêne faible ou absent se révèle favorable à la désignation de l'insecte comme utile. Ainsi, les individus rencontrés adhèrent d'autant plus à une désignation anthropocentrique du moustique qu'ils sont et/ou se sentent vulnérables vis-à-vis de l'insecte, vulnérabilité pouvant relever du simple confort ou bien appartenir à des considérations sanitaires. L'enquête révèle à ce titre que 51,25\% des personnes interrogées considèrent qu'en France métropolitaine, le moustique peut transmettre des maladies à l'homme. Une différence notable est à relever entre les terrains d'étude. Si 69 \% des habitants de la Camargue non démoustiquée considèrent que le moustique peut transmettre des maladies à l'homme en France métropolitaine, ils sont $55 \%$ en Petite Camargue démoustiquée et moins encore ( $40 \%$ ) pour le terrain alpin démoustiqué. Ces écarts peuvent en partie s'expliquer par la présence dans le delta du Rhône du virus du West Nile. En 2000, puis en 2003, plusieurs cas équins et humains se sont déclarés en Camargue et dans le Var (Zientara et al., 2004), dont la presse se fit d'autant plus l'écho que cet événement venait nourrir la controverse locale relative à la mise sur agenda politique d'un projet de démoustication de la commune. Pour l'ensemble des terrains, les «fièvres » sont les principales maladies imputées au vecteur moustique. Ces réponses peuvent s'appuyer en partie sur une connaissance vernaculaire, rappelant que les derniers cas connus de paludisme autochtone sont encore relativement proches dans la mémoire collective, puisqu'ils remontent à $1973^{10}$. D'autres réponses, évoquant notamment le sida ${ }^{11}$, ne résistent pas à l'objectivité de la médecine conventionnelle. Ces savoirs vernaculaires, peu ou prou en adéquation avec les savoirs scientifiques, articulent la persistance de peurs ancestrales et leur transfert à des références contemporaines.

Si l'influence du sentiment de gêne sur la qualification du moustique « utile» versus «nuisible » est commune à l'ensemble des terrains d'étude, elle s'observe toutefois dans des proportions très différentes selon la localité. La Camargue non démoustiquée se distingue, en effet, car même les habitants se déclarant gênés par les moustiques continuent majoritairement à qualifier l'insecte d'utile. Cependant, et c'est là une originalité locale, l'utilité du moustique en Camargue n'est pas seulement associée à son rôle dans l'équilibre écologique, mais aussi à une fonction de garde-frontière protégeant le delta du Rhône

\footnotetext{
10 Ces témoignages sont antérieurs à la découverte de trois cas soupçonnés de paludisme impliquant des moustiques de Corse, en septembre 2006 (Armengaud et al., 2007).

11 Il est prouvé que le sida ne peut pas être transmis par le moustique (Booth, 1987).
} 
et ses habitants, par l'inconfort qu'il procure, d'une éventuelle pression touristique et immobilière. Plus encore, le moustique peut servir de marqueur identitaire, un «vrai Camarguais » se devant de ne pas «craindre les moustiques » (Claeys-Mekdade, 2000 ; Claeys-Mekdade et Morales, 2002 ; Claeys-Mekdade et Nicolas, 2002). Cet usage symbolique du moustique garde-frontière et marqueur identitaire a aussi été observé dans le golfe du Morbihan, où la non-démoustication de ce territoire (qui connaît également une forte revendication identitaire) fait aussi controverse (Huneau, 2006). En revanche, il ne s'observe pas en Rhône-Alpes.

Outre ces effets cognitifs et identitaires, le regard porté sur le moustique se comprend aussi à la lumière des caractéristiques sociales et démographiques des locuteurs. Ainsi, quel que soit le terrain, les individus de moins de 60 ans qualifient davantage le moustique d'utile et, réciproquement, les plus de 60 ans sont plus enclins à le définir comme nuisible. De même, les individus bien dotés en capital culturel considèrent plus que les autres l'insecte comme utile, et inversement. Ainsi, la culture anthropocentrique de générations nées avant les trente glorieuses et/ou d'individus de milieux populaires, amenant ces derniers à qualifier le moustique de nuisible, semble céder du terrain à une considération biocentrique du monde animal portée par les nouvelles générations et/ou les individus ayant plus longtemps fréquenté les bancs de l'école et ses cours de sciences de la vie, véhiculant une nouvelle vision écosystémique de la nature (Lambert, 1999).

Enfin, chez près d'un tiers des personnes interrogées ${ }^{12}$, la frontière entre anthropocentristes et biocentristes est perméable, le paradoxe du nuisible/utile malmenant le dualisme anthropocentrisme/biocentrisme. Plutôt qu'une indécision, ces types de réponses en apparence contradictoires exprimeraient la cohabitation chez un même individu des référentiels anthropocentrique et biocentrique. En d'autres termes, cette figure du nuisible/utile souligne que le moustique serait nuisible à l'homme, qu'il pique, et utile à l'écosystème, dont il est un des maillons. Que faire dès lors de ce moustique nuisible/utile? Mettre un terme à ses nuisances, même au détriment de la qualité des écosystèmes qui l'abritent, ou bien le laisser vaquer à son utilité, même au détriment du confort cutané de l'être humain, voire de sa santé ? Face à ce dilemme, des réponses différentes et en évolution sont proposées selon le lieu, l'époque et le locuteur. Des demandes sociales plurielles, diffuses ou explicites, interpellent des politiques publiques qui se révèlent, dans ce domaine, réactives, voire anticipatrices.

\footnotetext{
${ }^{12}$ Soit 28 \% en Rhône-Alpes et 32 \% en Méditerranée.
}

\section{«Acrobaties taxinomiques » pour un enchevêtrement entre social et biologique}

Aux deux extrêmes de l'éventail de la demande sociale, s'opposent le tout-démoustication et le refus total de démoustication. De l'un à l'autre, s'enchaînent différentes postures glissant de l'anthropocentrisme au biocentrisme, par nuances successives mais aussi par la construction de compromis nés d'acrobaties taxinomiques plus ou moins solides. Dans les zones d'intervention des EID, la démoustication ne fait pas ou peu controverse. Pour autant qu'elle soit clairement perçue, la démoustication est considérée comme un acquis dont la remise en cause semble ne pas se poser. Plus encore, la démoustication a, pour beaucoup, un air de «main invisible». La démoustication, ses institutions, son financement et ses techniques sont en effet peu, voire mal connus de la majorité des habitants rencontrés ${ }^{13}$. Cet effet «main invisible » peut être lu comme une forme passive de compromis entre anthropocentrisme et biocentrisme, bâtie sur la non-visibilité de l'intervention humaine. On retrouve ici plus largement le schéma de la nature sauvage-aménagée, classiquement observé dans l'analyse des discours des usagers d'espaces «naturels » (Kalaora et Theys, 1992 ; Claeys-Mekdade et Jacqué, 2000), permettant d'articuler deux exigences potentiellement contradictoires, le désir de nature sauvage, d'une part, et, d'autre part, celui de sécurité et de confort. Tacitement ou explicitement, la demande sociale s'exprime en faveur des politiques de démoustication ${ }^{14}$. Minoritaires parmi les personnes rencontrées, les oppositions à la démoustication mettent en exergue la protection de la nature, du patrimoine et du territoire, ou bien des intérêts plus individuels, comme la tranquillité.

Toutefois, même les plus favorables à la démoustication expriment certaines réserves, sinon des inquiétudes. En effet, près de $60 \%$ des individus rencontrés soulignent la nocivité potentielle de la démoustication. Quel que soit le terrain, cette nocivité est d'abord associée aux effets de la démoustication sur la nature et, en second lieu, sur l'être humain (respectivement $59 \%$ et $26 \%$ ). Les individus rencontrés n'en tentent pas moins de rendre conciliables préoccupations biocentriques et demande en faveur d'une mise en place et/ou d'un maintien des politiques de démoustication. En effet, le recours à une projection spatialisée de la figure du moustique utile/nuisible permet d'échapper jusqu'à un certain point à ce paradoxe taxinomique et de résoudre le dilemme gestionnaire sous-jacent. Il y aurait, de ce point de vue,

\footnotetext{
13 En Camargue comme en Petite Camargue, plus de $40 \%$ des individus interrogés ne savent pas quel est l'organisme en charge de la démoustication. Ils sont $72 \%$ dans ce cas en Rhône-Alpes.

${ }^{14}$ Lors des entretiens semi-directifs, les interlocuteurs vivant en zones démoustiquées relèvent qu'il leur semble impossible de revenir à une situation de non-démoustication.
} 
des moustiques des villes, nuisibles et dont il convient de se débarrasser, et des moustiques des champs, ou plutôt des zones humides, utiles en tant qu'éléments de ces écosystèmes remarquables et qu'il conviendrait de ne pas perturber. Au cours des entretiens semi-directifs, ces catégories sont à plusieurs reprises mobilisées. Les réponses aux questionnaires vont aussi dans ce sens, en s'appuyant implicitement sur cette dichotomie ville/campagne. Ainsi, en Camargue, $82 \%$ des habitants interrogés se disent favorables à la démoustication des zones habitées, contre $66,5 \%$ pour l'ensemble du delta, zones naturelles incluses. Comme pour le rat de la fable de La fontaine, et d'Ésope avant lui, l'animal sauvage n'est pas à sa place dans la ville et prend le risque d'être dérangé; il serait en paix dès lors qu'il se tiendrait à sa place, c'est-à-dire à bonne distance de $\mathrm{l}^{\prime}$ homme ${ }^{15}$. La force, mais aussi les limites de cette acrobatie taxinomique vernaculaire résident dans ses fondements scientifiques. En effet, du point de vue biologique, on retrouve une classification des moustiques qui s'applique à cette séparation de l'espace entre ville et champs. Parmi les moustiques anthropophiles, certains, du genre Culex par exemple (Culex pipiens pipiens L.), ont su s'adapter aux mœurs citadines de leurs hôtes, s'accommodant de gîtes larvaires artificiels. Ainsi, ces moustiques se sont spécialisés à l'environnement urbain où ils trouvent profusion d'hôtes. En revanche, les Aedes demeurent inféodés aux espaces naturels. Tout se passe comme si ces moustiques, en compétition pour une ressource devenue limitante dans l'environnement rural (la densité d'hôtes), se partageaient l'espace et la ressource. Toutefois, la place socialement assignée aux moustiques des villes et aux moustiques des champs ne résiste pas au processus naturel de dispersion des insectes volants, capables de parcourir plusieurs kilomètres. En outre, l'étalement périurbain tend à brouiller les frontières entre «les villes et les champs ». Ainsi, la seule démoustication des villes ne permettrait pas ou peu de réduire l'exposition de leurs habitants aux piqûres, puisque l'Aedes, ce moustique des «champs », aidé par les vents qui le portent, s'invite sans difficulté à des repas en ville. Sans cesse, l'homme tente de cerner de ses taxinomies les êtres non humains et, sans cesse, ces derniers s'en échappent. On retrouve ici le caractère récalcitrant des objets naturels, souligné par Bruno Latour (1999). Dans ce contexte, les politiques publiques sont amenées à gérer ces demandes sociales plurielles et paradoxales et ces êtres non humains récalcitrants.

La mise en œuvre des politiques de démoustication au début des années 1960 était explicitement anthropocentrique. Protéger l'être humain, pour sa santé, son confort et son enrichissement, était en effet le premier objectif des EID. Dans l'esprit des trente glorieuses, la démoustication

\footnotetext{
15 Pour une analyse de « la place de l'animal », voir Staszak
} et al. (2002). se devait d'être efficace, au service du développement économique et de la modernisation de la nation. Or, singulièrement, cet objectif d'efficacité a rapidement amené à penser ce nouveau métier de "démoustiqueur ${ }^{16}$ » dans une perspective, sinon biocentrique, tout au moins intégrée.

Les premières expériences de démoustication « aveugle », visant les insectes adultes, ont rapidement été délaissées au vu de leur impact sur la nature. La lutte orchestrée en France par les EID n'a plus pour but d'éliminer les moustiques d'une région, mais plutôt de contrôler leurs populations pour limiter la nuisance qu'elles génèrent à un seuil «tolérable » par les riverains (Besnard et Gruffaz, 1999). Elle s'appuie en premier lieu sur l'entretien physique des milieux (drainage, action sur la végétation), pour limiter les facteurs de prolifération des moustiques. Ensuite, le suivi régulier des populations sur le terrain est réalisé, grâce à la cartographie écologique des milieux susceptibles de produire des moustiques (Rioux et al., 1967). Lorsque les populations larvaires sont importantes, leur contrôle par des insecticides s'impose, afin de prévenir de fortes densités d'adultes vulnérants. Ces insecticides étaient chimiques au début des campagnes : les EID ont, par exemple, utilisé des organo-phosphorés, comme la substance active téméphos, entre 1970 et 1989 en Rhône-Alpes et jusqu'en 2006 en Méditerranée. Le développement de politiques environnementales nationales et internationales et leur mise en application à travers l'appareil législatif ont progressivement amené les EID à intégrer des lois plus contraignantes concernant les usages des insecticides (directive européenne Biocide 98/8/CE, notamment). Les molécules à large spectre d'action ont ainsi progressivement cédé la place aux insecticides dits biologiques, comme le Bti (Bacillus thuringiensis var. israëlensis), plus sélectifs avec peu ou pas d'effets néfastes démontrés sur l'environnement (Becker et Margalit, 1993 ; Boisvert et Boisvert, 2000 ; Franquet et al., 2002). Ce bacille agit après ingestion, par des larves de Diptères, en libérant des toxines protéiques qui perforent l'épithélium intestinal et conduisent à leur mort. Cependant, les traitements au Bti coûtent plus cher, du fait du prix de vente du produit actif, mais aussi des méthodes d'épandage qu'il nécessite (plus grand recours aux moyens aériens) ${ }^{17}$. En outre, l'utilisation d'un seul et unique insecticide peut augmenter le risque de développement de résistances

\footnotetext{
16 «Démoustiqueur » est l'appellation communément utilisée en Camargue et en Petite Camargue par les habitants et les acteurs locaux.

17 Sur le littoral méditerranéen, la végétation dense de certains gîtes fait obstacle à son épandage aérien, et les larves n'y sont sensibles qu'en début de vie, pendant une courte période, laissant peu de temps au gestionnaire pour agir lorsque l'insecticide employé est le Bti. (Sources : entretiens semi-directifs, responsables EID Méditerranée.)
} 
par les moustiques (Casida et Quistad, 1998). Toutefois, des populations de moustiques résistantes au Bti n'ont à ce jour jamais été signalées, du fait de la complexité des toxines de la bactérie. Enfin, le pendant de la plus grande sélectivité de cet insecticide biologique serait une efficacité moindre. Ces considérations, mises en avant par les EID lors de leurs débats avec le ministère de l'Écologie et du Développement durable, soulignent la difficulté à trouver un compromis entre préoccupations écologiques, économiques, sociales et politiques, face à des êtres humains et non humains aux caractéristiques plurielles et changeantes.

\section{Conclusion}

Dynamiques sociales et naturelles des moustiques se croisent, soulevant considérations culturelles, politiques, juridiques, économiques, sanitaires et biologiques. Pour comprendre l'évolution du traitement social du moustique, il a été indispensable de comprendre l'évolution du traitement social des zones humides. Des miasmes du marais à éradiquer à la zone humide "naturelle » à protéger, de l'anthropocentrisme au biocentrisme, le moustique lui-même n'échappe pas à ce glissement taxinomique, l'opération de redéfinition symbolique de son habitat rejaillissant sur le statut de l'insecte anthropophile. Toutefois, les dynamiques sociales et biologiques n'ont pas la linéarité qu'il est parfois tentant de leur prêter. La figure paradoxale du moustique utile/nuisible peut aussi bien être révélatrice d'une phase transitoire qui dure, à l'instar de la «seconde modernité » d'Ulrich Beck (1992), ou bien d'une forme de compromis sur le mode de la «modernisation écologique » soulignée par Arthur Mol (1996). Telle est la force du social : rendre tenables la pluralité et le paradoxal, plus ou moins durablement, plus au moins solidement, face à la force du biologique qui sans cesse échappe aux taxinomies et aux actions humaines. Le traitement au Bti en France métropolitaine constitue à ce jour un compromis socio-naturel ${ }^{18}$, en tant qu'équilibre (forcément provisoire, forcément fragile) entre le social et le biologique. Ce compromis tient à l'objectivation imparfaite, voire illusoire, de ce que les gestionnaires appellent le seuil de tolérance.

De l'évolution des politiques de gestion des zones humides et de leurs moustiques, se dégagent quelques paradoxes. Si les politiques anthropocentriques d'assèchement des marais cèdent la place à des politiques biocentriques de conservation, en revanche, les politiques anthropocentriques de démoustication se développent au moment où l'insecte ne représente plus en France métropolitaine de risque sanitaire significatif, lui donnant

\footnotetext{
18 L'expression «socio-naturelle » fut initialement proposée
} par Charvolin (1993). explicitement les traits de l'utilitarisme économique, appelant deux nouveaux paradoxes. En premier lieu, c'est la recherche de l'efficacité et de la rentabilité de la démoustication qui contribue à développer très tôt des méthodes dites intégrées, c'est-à-dire se rapprochant sensiblement de postures biocentriques. En second lieu, l'utilitarisme économique de la démoustication s'oppose dans ses fondements mêmes au biocentrisme, tout en retirant des intérêts directs de la protection de la nature. La démoustication, cette offre de bien-être pour attirer et retenir touristes et main-d'œuvre, trouve une alliée dans les externalités positives que procure la protection de la nature productrice/conservatrice de paysages remarquables. Ainsi, même poussé dans la radicalité de l'écocentrisme, l'anthropocentrisme peut trouver quelques arrangements avec le biocentrisme, à moins que ce dernier ne soit qu'un avatar paradoxal de l'anthropocentrisme lui-même. Car, in fine, qu'il exploite ou bien qu'il protège la nature, c'est l'homme qui définit le télos, cet idéal de nature à atteindre par lui, mais aussi pour lui, tout autant, sinon plus, que pour elle.

\section{Références}

Allard, P., 1999. Les politiques des grands aménagements au $\mathrm{XIX}^{\mathrm{e}}$ siècle et le rôle des ingénieurs. Communication au séminaire SEAH (Systèmes écologiques et action de l'homme), Arles, janvier.

Armengaud, A., Legros, F., D'Ortenzio, E., Quatresous, I., Barre, H., Houze, S., Valayer, P., Fanton, Y., Schaffner, F., 2007. Survenue en Corse d'un cas de paludisme autochtone à Plasmodium vivax, France, août 2006, Bulletin épidémiologique hebdomadaire, 25-26, 231-233.

Bata, P., Péan, A., Guillemet, D., Péret, J., Soubiran, J.-R. (Eds), 2002. Aux rives de l'incertain : histoire et représentation des marais occidentaux du Moyen Âge à nos jours, Paris, Somogy.

Beck, U., 1992. Risk Society : Towards a New Modernity, London, SAGE Publications.

Becker, N., Margalit, J., 1993. Use of Bacillus thuringiensis israelensis against mosquitoes and blackflies, in Entwistle, P.F., Cory, J.S., Bailey, M.J., Higgs, S. (Eds), Bacillus thuringiensis, an Environmental Biopesticide: Theory and Practice, New York, Wiley, 147-170.

Besnard, G., Gruffaz, R., 1999. Préservation de l'environnement et lutte contre des espèces vulnérantes pour l'homme : inventaire et cartographie des invertébrés comme contribution à la gestion des milieux naturels français, Actes du séminaire tenu à Besançon les 8, 9 et 10 juillet 1999, Paris, Publications scientifiques du Muséum national d'histoire naturelle, 171-173.

Boisvert, M., Boisvert, J., 2000. Effects of Bacillus thuringiensis var. israelensis on target and nontarget organisms : A review of laboratory and field experiments, Biocontrol Science and Technology, 10, 5, 517-561.

Booth, W., 1987. AIDS and insects, Science, 237, 4813, 355-356.

Chapuis, J.-L., Barre, V., Barnaud, G. (Eds), 2001. Recréer la nature: réhabilitation, restauration et création d'écosystèmes. Document de synthèse, Programme national de recherche du ministère de l'Aménagement du territoire et de l'Environnement, Paris. 
Charvolin, F., 1993. L'Invention de l'environnement en France : chronique anthropologique d'une institutionnalisation, Paris, La découverte.

Claeys-Mekdade, C., 2000. Les Conflits d'aménagement : rapports à la "nature » et rapports sociaux. La Camargue, un cas révélateur. Thèse de doctorat en sociologie, Université de Provence, Aix-en-Provence.

Claeys-Mekdade, C., 2003. Le Lien politique à l'épreuve de l'environnement : expériences camarguaises, Bruxelles, P.I.E-Peter Lang.

Claeys-Mekdade, C., Jacqué, M., 2000. Le massif forestier de la Clape : nature de proximité et nature pittoresque? Étude de fréquentation auprès des usagers, Forêt méditerranéenne, XXI, $1,19-24$

Claeys-Mekdade, C, Corsand, L.M., Nicolas, L., SchleyerLindenmann, A., 2002. Être ou ne pas être entre les deux bras du Rhône : identité(s) camarguaise(s) aujourd'hui, Faire Savoirs, 2, 23-35.

Claeys-Mekdade, C., Morales, A., 2002. Moustiques et démoustication : une enquête sociologique auprès des Arlésiens et des Camarguais. Contrat PNRC/IMEP - DESMID, rapport final.

Claeys-Mekdade, C., Nicolas, L., 2002. Être ou ne pas être démoustiqué, enquêtes ethnologique et sociologique comparatives : Camargue et Petite Camargue. Contrat DESMIDEID, programme européen LIFE Environnement (LIFE 99env/F/000489), rapport d'enquête.

Corbin, A., 1986. Le Miasme et la jonquille : l'odorat et l'imaginaire social, XVIII ${ }^{e}$ XIX ${ }^{e}$ siècles, Paris, Flammarion.

Franquet, E., Fayolle, S., Hudin, S., 2002. Quatrième rapport intermédiaire sur l'étude d'impact d'un éventuel traitement au B.T.I. sur le territoire du Parc naturel régional de Camargue. Contrat PNRC/UDESAM.

Godelier, M., 1984. Le Matériel et l'idéel, Paris, Fayard.

Huneau, V., 2006. Enquête et analyse sociologique d'une zone non démoustiquée du Morbihan : Séné, Theix et Saint-Armel. Rapport d'enquête, Entente interdépartementale pour la démoustication du littoral Atlantique.
Kalaora, B., Theys, J. (Eds), 1992. La Terre outragée : les experts sont formels, Paris, Autrement.

Lambert, S., 1999. Quand l'écologie et la biologie s'appelaient histoire ou sciences naturelles. Application aux animaux utiles ou nuisibles, Le Courrier de l'environnement de l'INRA $38,23-40$

Latour, B., 1999. Les Politiques de la nature, ou Comment faire entrer les sciences en démocratie, Paris, La Découverte.

Mol, A.P.J., 1996, Ecological modernization and institutional reflexivity. Environmental reform in the late modern age, Environmental Politics, 5, 2, 302-323.

Pelen, J.-N., 1987. Le Pays d'Arles par ses gens : sentiment d'appartenance et référents d'identité. Rapport pour la Mission du patrimoine ethnologique, Paris.

Picon, B., 1978. L'Espace et le temps en Camargue, Arles, Actes Sud.

Rioux, J.-A., Crozet, H., Corre, J.-J., Simonneau, P., 1967. Les bases phyto-écologiques de la lutte anticulicidienne. Cartographie des biotopes larvaires, ses applications opérationnelles dans le Midi méditerranéen, Annales de parasitologie humaine et comparée, 42, 6, 665-680.

Rodhain, F., Perez, C., 1985. Précis d'entomologie médicale et vétérinaire, Paris, Maloine.

Schaffner, F., 1998. A revised checklist of the French Culicidae, European Mosquito Bulletin, 2, 1-9.

Sérandour, J., Girel, J., Boyer, S., Ravanel, P., Lemperiere, G., Raveton, M., 2007. How human practices have affected vector-born diseases in the past : A study of malaria transmission in Alpine Valleys, Malaria Journal, 6, 115.

Staszak, J.F., Blanc, N., Cohen, M. (coord.), 2002. « La place de l'animal ", dossier thématique, Espaces et sociétés, 110-111.

Willott, E., 2004. Restoring nature, without mosquitoes?, Restoration Ecology, 12, 22, 147-153.

Zientara, S., Durand, B., Mailles, A., 2004. Lutte concertée contre l'infection à virus West-Nile, Épidémiologie et santé animale, $46,63-66$

Reçu le 21 novembre 2007. Accepté le 5 février 2009. 